\title{
Targeting Metabolic Plasticity in Breast Cancer Cells via Mitochondrial Complex I Modulation
}

\author{
Qijin $\mathrm{Xu}, \mathrm{PhD}^{1}$, Eva Biener-Ramanujan, $\mathrm{PhD}^{2}$, Wei Yang, $\mathrm{PhD}^{1,3}$, and V Krishnan \\ Ramanujan, PhD ${ }^{1,3,4,5,{ }^{*}}$ \\ ${ }^{1}$ Metabolic Photonics Laboratory, Department of Surgery, Cedars-Sinai Medical Center, 8700 \\ Beverly Blvd., Los Angeles, CA 90048, USA \\ ${ }^{2}$ Department of Medicine, Cedars-Sinai Medical Center, 8700 Beverly Blvd., Los Angeles, CA \\ 90048, USA \\ ${ }^{3}$ Department of Biomedical Sciences, Cedars-Sinai Medical Center, 8700 Beverly Blvd., Los \\ Angeles, CA 90048, USA \\ ${ }^{4}$ Biomedical Imaging Research Institute, Cedars-Sinai Medical Center, 8700 Beverly Blvd., Los \\ Angeles, CA 90048, USA \\ ${ }^{5}$ Samuel Oschin Comprehensive Cancer Institute, Cedars-Sinai Medical Center, 8700 Beverly \\ Blvd., Los Angeles, CA 90048, USA
}

\section{Abstract \\ Purpose-Heterogeneity commonly observed in clinical tumors stems both from the genetic diversity as well as from the differential metabolic adaptation of multiple cancer types during their struggle to maintain uncontrolled proliferation and invasion in vivo. This study aims to identify a potential metabolic window of such adaptation in aggressive human breast cancer cell lines.}

Methods-With a multidisciplinary approach using high resolution imaging, cell metabolism assays, proteomic profiling and animal models of human tumor xenografts and via clinicallyrelevant, pharmacological approach for modulating mitochondrial complex I function in human breast cancer cell lines, we report a novel route to target metabolic plasticity in human breast cancer cells.

Results-By a systematic modulation of mitochondrial function and by mitigating metabolic switch phenotype in aggressive human breast cancer cells, we demonstrate that the resulting

\footnotetext{
*Corresponding author: V Krishnan Ramanujan, PhD, Assistant Professor, Metabolic Photonics Laboratory, Departments of Surgery \& Biomedical Sciences, Cedars-Sinai Medical Center, 8700 Beverly Blvd., Davis 6067, Los Angeles, CA 90048, USA Tel: $(+1)$ 310-423-7666, Ramanujanv@csmc.edu.

Qijin Xu, PhD, Metabolic Photonics Laboratory, Department of Surgery, Cedars-Sinai Medical Center, 8700 Beverly Blvd., Los Angeles, CA 90048, USA, XuQ@cshs.org

Eva Biener-Ramanujan, PhD, Inflammatory Bowel and Immunobiology Research Institute, Department of Medicine, Cedars-Sinai Medical Center, 8700 Beverly Blvd., Los Angeles, CA 90048, USA, Ramanujane@cshs.org

Wei Yang, PhD, Departments of Surgery \& Biomedical Sciences, Cedars-Sinai Medical Center, 8700 Beverly Blvd., Los Angeles, CA 90048, USA, YangWXX@cshs.org

Conflict of Interest

The authors declare that they have no conflict of interest.

Ethical Standards

The authors declare that all the experiments described in this study comply with current laws of the United States of America.
} 
metabolic adaptation signatures can predictably decrease tumorigenic potential in vivo. Proteomic profiling of the metabolic adaptation in these cells further revealed novel protein-pathway interactograms highlighting the importance of antioxidant machinery in the observed metabolic adaptation.

Conclusions-Improved metabolic adaptation potential in aggressive human breast cancer cells contribute to improving mitochondrial function and reducing metabolic switch phenotype -which may be vital for targeting primary tumor growth in vivo.

\section{Keywords}

Metabolic Switch; Mitochondrial Complex I; Breast cancer; Metabolic Plasticity; Catalase; Antioxidant Pathway

\section{Introduction}

Metabolic switch or aerobic glycolysis phenotype is currently understood as a common denominator in multiple breast cancer subtypes.[1-9] By definition, this phenotype implies glycolytic upregulation even in the presence of oxygen thereby causing a bioenergetic shift ('metabolic switch") from mitochondrial to predominantly glycolytic pathway. Increased glycolytic activity has proliferative advantages (e.g., biosynthesis of nucleic acids, fatty acids etc.,) as well as pro-angiogenic effects (e.g., VEGF upregulation).[10] It is not clear if mitochondrial dysfunction is a necessary condition for observing metabolic switch phenotype in transformed cells as proposed in Warburg's original hypothesis.[9] In an effort to obtain clarity on this question, we recently demonstrated that while metabolic switch phenotype can be induced by mitochondrial dysfunction, it is also possible to partially reverse this phenotype in situations where mitochondrial dysfunction is only moderate.[11] This earlier study led to the hypothesis that the connection between mitochondrial dysfunction and metabolic switch phenotype in cancer cells may not always be permanent as originally understood. In other words, cancer cells may exhibit a "metabolic plasticity" regime under certain conditions - which may further be amenable for targeting tumor control in vivo. In order to test this idea in a preclinical setting, we used pharmacological modulation of mitochondrial complex I function in a few candidate breast cancer cells. Mitochondrial complex I is the first committed step in the mitochondrial electron transport chain and is also the gate keeper between glycolytic/TCA substrate metabolism and mitochondrial electron transport cascade that ultimately determines cellular ATP generation. [12] The rationale for this approach is that by culturing the cancer cells under the long-term selection pressure of mitochondrial complex I modulation, cancer cells might display reprogrammed energy metabolism and plausibly, an altered cancer cell phenotype via adaptive metabolism. By generating representative, isogenic human breast cancer cells via mitochondrial complex I modulation, we studied the effects of long-term metabolic adaptation in these cells. The cells that showed a consistent increase in adaptation potential displayed a robust enhancement in mitochondrial function, a significant decrease in metabolic switch phenotype as well as profound reduction in cell proliferation in vitro as well as in tumor xenografts in vivo. A global proteomic profiling in these adaptive cells further indicated an intricate interplay between cancer-related pathways, cell cycle proteins 
and antioxidant pathways that collectively contributed to a reduction in tumorigenic potential in vivo. Together, our results point out to an attractive strategy for targeting the metabolic plasticity regime for tumor control in vivo.

\section{Materials and Methods}

A complete summary of the methods can be found in Supplemental data document.

\subsection{Cell Culture \& Metabolic Characterization}

The non-transformed MCF10A and the human breast cancer cell lines (MDA-MB-231, MDA-MB-468 and MDA-MB-453) were cultured and maintained as per the ATCC guidelines. In order to generate isogenic cancer cell lines with selective adaptation to mitochondrial complex I modulation, we cultured the two cancer cell lines, MDA231 and MDA453, in the presence of $100 \mathrm{nM}$ of mitochondrial complex I inhibitor, rotenone continuously for 20 generations after which the rotenone selection pressure was removed. No observable toxicity was noticed during this procedure. The following notation will be used in this paper : (A) Parental cells: 231-P \& 453-P; (B) Adaptive cells : 231-100R \& $453-100 \mathrm{R}$ where $\mathrm{P}$ refers to Parental and 100R refers to (100nM Rotenone) selection pressure. Glucose uptake, mitochondrial membrane potential status, hydrogen peroxide and superoxide levels in live cells were measured by flow cytometry analysis in live cells respectively by labeling with $100 \mu \mathrm{M} 2 \mathrm{NBDG}, 200 \mathrm{nM}$ TMRM, 2.5 $\mu \mathrm{M}$ DCFDA and $2.5 \mu \mathrm{M}$ MitoSox Red. All the reagents were obtained from Life Technologies, Grand Island, NY, USA. Lactate measurements and mitochondrial respiration measurements were carried out as described earlier.[11] Superoxide Dismutase (SOD) activity assay: SOD activity assays were performed using SOD Assay Kit (ScienCell Cat \#8198, Carlsbad, CA) following manufacturer's instructions with volume scaling-down for 96-well plate. Mitochondrial complex I activity was measured as described earlier.[11] A modified Amplex red assay (Catalog \# A22188, Life Technologies (Invitrogen), Grand Island, NY, USA) was used to estimate catalase activity in breast cancer cells. Briefly this assay relies on the enzymatic activity of hydrogen peroxide/peroxidase pair measured by the Amplex Red reagent (10acetyl-3-7-dihydroxyphenoxazine). In the presence of peroxidase, the Amplex Red reagent reacts with $\mathrm{H}_{2} \mathrm{O}_{2}$ in a 1:1 stoichiometry to produce the red-fluorescent oxidation product, resorufin $(571 \mathrm{~nm}$ excitation; $585 \mathrm{~nm}$ emission. Protein estimation was carried out by either standard SDS-PAGE or blue-native PAGE protocols (Supplemental Section). High resolution immunofluorescence images were obtained in a Leica confocal microscope platform (fixed tissues and cells) or in a Leica two-photon excitation microscope platform (3D live cell cultures) as described elsewhere.[13,14,11] All the data presented in this paper are mean \pm S.E from at least three independent experiments unless otherwise mentioned. Statistical significance was estimated based on Student's t-test $(\mathrm{p}<0.05)$.

\section{2: Animal Studies}

Athymic mice (nu/nu, 8 weeks old, Charles River Laboratories, Wilmington, MA) were implanted subcutaneously with parental $231-\mathrm{P}$ and adaptive $231-100 \mathrm{R}$ cells $\left(1 \times 10^{6}\right.$ cells per mouse) on each side of the flank in groups of five animals. After 7-8 weeks postimplantation, animals were sacrified and final tumor sizes were measured using a caliber and 
weighed before tissue processing. Xenograft tumors were processed with formalin before being embedded in paraffin. Tissue sections were then processed by standard protocol either for hematoxylin \& eosin staining or immunofluorescence staining [monoclonal antibodies probing Ki67 or SOD2 or catalase] All animal experiments were performed in strict accordance with the Institutional Animal Care and Use Committee guidelines.

\subsection{Mass Spectrometry and Proteomics}

Mass-spectrometry based proteomics analysis was performed in the parental (231-P) and adaptive (231-100R) cells cultured as described earlier.[15] Stable isotope labeling of amino acids (SILAC) labeling involved growing the cells for at least six doublings in arginine- and lysine-depleted DMEM medium supplemented with 10\% (v/v) dialyzed FBS (Invitrogen, Grand Island, NY), $100 \mathrm{U} / \mathrm{mL}$ penicillin and $100 \mu \mathrm{g} / \mathrm{mL}$ streptomycin, and L-arginine (Arg0) and L-lysine (Lys0) (Pierce, Rockford, IL) for 231-P cells or ${ }^{13} \mathrm{C}_{6}{ }^{15} \mathrm{~N}_{4}$-L-arginine (Arg10) and ${ }^{13} \mathrm{C}_{6}{ }^{15} \mathrm{~N}_{2}$-L-lysine (Lys8) (Cambridge Isotope Laboratories, Andover, MA) for 231-100R cells. Proteins extracted from SILAC-labeled 231-P (light isotope) and 231-100R (heavy isotope) cells were mixed in equal amounts. Protein digestion was performed using the filter-aided sample preparation (FASP) method with slight modifications.[16] Tryptic peptides were collected and analyzed by an EASY-nLC1000 LC system connected to an LTQ Orbitrap Elite mass spectrometer (Thermo Scientific). For each fraction, $10 \mu \mathrm{L}$ peptide solution was loaded onto a $2 \mathrm{~cm}$ Acclaim precolumn ( $75 \mu \mathrm{m}$ inner diameter, PepMap $\mathrm{C}_{18}, 3$ $\mu \mathrm{m}$ particle size) connected to a $50 \mathrm{~cm}$ EASY-Spray column $(75 \mu \mathrm{m}$ inner diameter, PepMap $\mathrm{C}_{18}, 3 \mu \mathrm{m}$ particle size). MS data were acquired in a data-dependent strategy selecting the fragmentation events based on the precursor abundance in the survey scan (400-1400 Th). For protein identification and quantification, raw mass spectrometric data were analyzed with MaxQuant software (version 1.3.0.5) in combination with the Andromeda search engine.[17,18] The parameters were set as follows. Oxidation (M), acetyl (Protein N-term), deamindation (NQ), and gln->pyro-glu were set as variable modification; carbamidomethyl (C) was set as fixed modification; Uniprot_Human database was used for database searching; all other parameters were default. False discovery rates for protein and peptide identifications were both set at 0.01 . Differentially expressed proteins (DEPs) were identified using Persus (v1.4.0.0) (http://www.perseus-framework.org/). Firstly, a list of quantified proteins were generated by filter the ProteinGroups.txt file to remove 1) all proteins identified from the reverse sequence database and contaminating protein database, 2) all proteins identified based on one peptide, and 3) all proteins quantified based on one measurement. Subsequently, significance B, which is an outlier probability calculated on protein subgroups obtained by intensity binning for each protein was computed. Proteins with significance B $<0.05$ were accepted as DEPs. Network modeling was performed using Ingenuity Pathway Analysis (Ingenuity).

\section{Results}

\subsection{Breast cancer cells display distinct metabolic status and sensitivity to mitochondrial complex I modulation}

An initial screening of three representative human breast cancer cell lines for determining the basal mitochondrial status revealed a wide variation in oxidative phosphorylation 
(OxPhos) subunit composition (Figure 1a). Less aggressive MDA453 cells and nontransformed MCF10A cells showed a similar pattern while the metastatic MDA231 cells showed a significant reduction in the mitochondrial OxPhos subunit expression. Figure $1 \mathrm{~b}$ shows significant reduction in oxygen consumption rate in both the breast cancer cell lines. To test the amenability of these cells to mitochondrial complex I modulation, mitochondrial complex I enzyme activity was measured in the presence of increasing concentrations of a known mitochondrial complex I inhibitor, rotenone (Figure 1c). MDA231 cells showed the highest sensitivity while MDA453 cells showed only a modest sensitivity to rotenone in comparison to that of non-transformed MCF10A cells. Another metatstatic cell line, MDA468 cells displayed severe reduction in expression levels of the OxPhos subunits (Figure 1a) as well as in oxygen consumption rate (data not shown). Since the main premise of this study was to evaluate the metabolic adaptation potential via mitochondrial reprogramming, MDA468 cell line was excluded from further studies. Supporting metabolic measurements addressing the effects of acute rotenone treatment on the three cell lines further confirmed that MDA231 cells demonstrated statistically significant changes in glucose uptake as well as free radical generation suggesting the viability of this pharmacological route to mitochondrial complex modulation (Figure 1d).

\subsection{Long-term selection with rotenone elicits differential metabolic adaptation signatures in breast cancer cells}

Having identified two breast cancer cell lines with distinct metabolic status as well as differential sensitivity to rotenone, we then set out to determine the long-term adaptation signatures in these cells that can impact their tumorigenic potential. Figure 2a shows a schematic for generating adaptive breast cancer cells. Briefly, this illustrates that upon long term complex I modulation, the viable breast cancer cells may comprise of insensitive (i.e., resistant to complex I modulation) population or sensitive population. In the former case the tumorigenic potential of the parental breast cancer cells may not necessarily be altered. On the other hand, if the complex I modulation induces an adaptive response in breast cancer cells so as to enhance their mitochondrial function and to decrease their tumorigenic potential- then we will have in effect, targeted the "metabolic plasticity" regime that is distinctly different from the resistance phenotype. According to this scheme, the parental breast cancer cells (231-P \& 453-P) were continuously cultured in the presence of complex I inhibitor (100nM rotenone). After 20 generations in the presence of rotenone selection pressure, the adaptive breast cancer cells (231-100R \& 453-100R) thus generated were subsequently cultured in normal culture conditions without rotenone. No global genetic differences were observed between the parental and the adaptive breast cancer cells (Supplemental Fig.S1). The first striking result was that the adaptive 231-100R cells displayed a significant reduction $(\sim 40 \%)$ in cell growth and cell cycle parameters in comparison to the parental 231-P cells (Figure 2b-2c). Besides, there was a significant reduction in both lactate generation rate (metabolic switch phenotype measure) in 231-100R cells as compared with the parental 231-P cells (Figure 2d). In contrast, the adaptive 453-100R cells did not show any significant change in cell growth parameters or in mitochondrial enhancement- in comparison to the parental 453-P cells. Quantitative qPCR (mRNA) measurements revealed significant changes in mitochondrial complex I electron transport chain subunits in both 231-100R and 453-100R cells (Figure 3a \& 3b). To further 
illustrate that the observed adaptive features in 231-100R cells are not transient, we compared the qPCR profiles of mitochondrial complex I subunits in 231-100R cells - in the presence of rotenone selection pressure and about 20 generations after removing the rotenone selection pressure. As can be seen from Figure 3a, the mitochondrial biogenesis profiles are almost identical in 231-100R cells with and without the selection pressure suggesting that the long term metabolic adaptation to mitochondrial complex I modulation contributes to more permanent phenotypic changes both at the transcriptional and protein level (Figure $3 c \& 3 d$ ). This was further confirmed by gene expression array analysis. (Supplemental Figure S2). Figures $4 \mathrm{a}-\mathrm{c}$ demonstrate significant enhancement in mitochondrial respiration and mitochondrial complex I activity and altered metabolic features (glucose uptake, hydrogen peroxide generation and superoxide generation) that are representative of the whole cell populations. More intriguingly the slow growing 231-100R cells had significantly lower levels of hydrogen peroxide generation but not the mitochondrial superoxide levels - suggesting non-transient, functional improvement in metabolic status in adaptive $231-100 \mathrm{R}$ cells.

\subsection{Metabolic adaptation-induced reduction in tumor cell proliferation is retained in tumor xenografts in vivo}

The observed reduction in cell growth was further confirmed in three-dimensional cell cultures grown in Mammocult medium which is a validation platform for anchorage independent growth as well as stemness. The parental 231-P cells showed anchorageindependent 3D growths with sizes ranging from about 50 to $250 \mu \mathrm{m}$ while the adaptive 231-100R cells did not grow 3D cell growths larger than $\sim 50 \mu \mathrm{m}$ as confirmed by twophoton excitation fluorescence imaging (Figure 5a-c). MDA453-P and 453-100R cells did not show any significant difference in their 3D growth potential under the same conditions (data not shown). In tumor xenograft models, the adaptive 231-100R cells displayed a consistently smaller tumor volumes as well as reduced tumor cell proliferation (Ki67 staining) than those with the parental 231-P cells as summarized in Figures 5d-g. A similar animal injection protocol with 453-P and 453-100R cells did not yield observable tumor xenografts in accordance with the earlier reports of non-tumorigenic nature of these cell lines.

\subsection{Proteomic profiling of adaptive cancer cells renders novel pathways supporting the metabolic adaptation}

In an attempt to understand the whole cell ramifications of the observed metabolic adaptation, we set out to determine the global proteomic signatures of adaptive metabolism in 231-100R cells that contributed to the tumor growth reduction. Mass spectrometry analysis of SILAC labeled 231-P (Light) and 231-100R (Heavy) cells (whole cell protein lysates as described in Supplemental data) showed that about 390 proteins were significantly different between the two cell lines studied (Figure 6a). Table 1 lists a comprehensive list of canonical pathways that were significantly altered in the adaptive $231-100 \mathrm{R}$ cells. Out of multiple pathway interaction scenarios, we arrived at one plausible interactogram (Figure 6b) that encompasses three major pathways namely, (a) cell cycle/growth (b) Redox/ROS/ Antioxidants and (c) breast cancer specific proteomic profiles. The rationale for this starting point was that we observed significant phenotypic changes in terms of cell growth/cell cycle 
in adaptive 231-100R cells. Furthermore the adaptive 231-100R cells displayed reduced hydrogen peroxide levels (Figure 4c) that might be indicative of selective antioxidant machinery that might be more functional in these cells. An earlier proteomics study in the mitochondrial fractions of these two cell lines had shown a significant upregulation of antioxidant enzyme, catalase in the adaptive 231-100R cells (data not shown) - adding support to this reasoning. With this motivation, we compiled a short list of protein signatures that were statistically significant in the adaptive 231-100R cells as summarized in Figure $6 \mathrm{~b}$. Since, within the scope of this study, it is impractical to validate all these potential protein signatures, we present our preliminary results validating one strong candidate marker namely, catalase.

\subsection{Validation of a potential antioxidant pathway in the adaptive 231-100R cells}

Recently we had reported that in cases of mild mitochondrial dysfunction, it was possible to reverse the metabolic switch phenotype by antioxidants (superoxide dismutases, SOD) that target mitochondrial superoxide levels in transformed cells.[11] Since adaptive 231-100R cells showed increased mitochondrial function and decreased metabolic switch phenotype, we hypothesized that a similar antioxidant route might be operational in these cells. However we did not detect any significant differences in SOD activity between the parental and adaptive cancer cells. On the other hand, we did detect statistically significant changes in another antioxidant enzyme (catalase) that is responsible for scavenging hydrogen peroxide free radicals in mammalian cells (Figure 7a-e). These results were further confirmed by immunoblotting where mitochondrial catalase was significantly higher in 231-100R cells as compared to the parental 231-P cells (Fig. 7f). Similar difference in catalase expression was observed in tissue slices obtained from the tumor xenografts generated by injecting 231-P \& 231-100R cells (Figure 8a-b) in accordance with the in vivo results in Section 3.3. To test if the observed increase in catalase expression/activity has a role in mediating the adaptation-induced growth reduction in $231-100 \mathrm{R}$ cells, both the parental and modified cells were cultured in the presence of a known pharmacological inhibitor of catalase (3-aminotriazole, 3AT).[19,20] Inhibition of catalase in 231-100R cells indeed contributed to an increase in hydrogen peroxide generation as well as an increase in cell proliferation - comparable to the parental 231-P cells. These preclinical observations were further validated in clinical breast cancer specimens in an effort to determine the clinical significance of catalase expression related to tumor growth. Immunofluorescence imaging in tissue microarrays of human breast tissue samples (normal, hyperplasia and invasive ductal carcinoma) showed a statistically significant loss of catalase expression in invasive breast carcinomas in comparison with the normal and non-invasive hyperplasia breast tissue specimens (Figure 9 and Supplemental Figures S4 \& S5). Together these data point out to a critical role that antioxidant machinery might play in mediating the metabolic adaptation potential in breast cancer cells.

\section{Discussion}

Despite the advances in our understanding of the genetic paradigm in carcinogenesis, cancer treatment regimens are still dominated by non-genetic, pharmacological approaches such as chemotherapy and hormone therapy.[7,3,5,21-27] It will be valuable to have an additional 
layer of targeting tumor metabolism in order to make the cancers amenable to reduction in primary tumor reduction and/or to therapeutic intervention. In this context, we have demonstrated here a novel route to modulate the metabolic plasticity in human breast cancer cells via mitochondrial complex I function. In transformed cells showing strong metabolic switch phenotype, glycolytic intermediates have been shown to play a significant role in supporting biosynthetic routes in cancer cells.[10] It is therefore conceivable that the cancer cells, during their adaptation in host environment - could induce this metabolic switch from mitochondrial to predominantly glycolytic pathways in order to support their proliferative demands even before hypoxia state is reached. The present study supports our premise that breast tumors are heterogeneous not only in their genetic profiles but also in their metabolic adaptation characteristics. On one hand, cancer cells can adapt so as to increase their survival and to evade apoptosis - as in the case of developing chemoresistance. On the other hand, cancer cells can also adapt so as to downplay their tumorigenic potential. The data shown here add support to this novel metabolic "window of opportunity" for modulating tumorigenic potential in human breast cancer cells. We have demonstrated that aggressive human breast cancer cells can be systematically reprogrammed to yield adaptive isogenic cell populations with significantly enhanced mitochondrial function and a concomitant reduction in metabolic switch phenotype. In accordance with a recent report identifying mitochondrial complex I as critical for defining the aggressive phenotype in breast cancer cells via NADH/NAD ${ }^{+}$balance [12], our results further validate the central importance of mitochondrial complex I function in breast cancer adaptation in vivo. Proteomic profiling of the adaptive cells revealed multiple metabolic alterations such as serine/glycine metabolism, aryl hydrocarbon receptor signaling as well as glutathione mediated redox/ROS metabolism. We believe that these metabolic alterations collectively determine the less tumorigenic phenotype in the adaptive cancer cells thereby illustrating a metabolic plasticity regime in these cells. The adaptive breast cancer cells further showed a global interplay at the proteomic level between classical cancer-related markers (e.g., TP53), antioxidant machinery (e.g., CAT, GPx) and cell cycle pathways. By identifying the distinct metabolic plasticity in windows in multiple cancer cell types, we envision a potentially unified metabolic metrics of cancer cell adaptation in vivo. This knowledge could offer valuable metabolic biomarkers in addition to the repertoire of currently known genetic markers. Validation studies of one of the candidate markers (catalase) identified in proteomics study, revealed that catalase was critical in mediating the reduction in cell proliferation in vitro and in vivo,. It is plausible that mitochondrial complex I modulation and the concomitant adaptation of the cells do activate a common antioxidant machinery in the adaptive cells. Since catalase was earlier reported to influence tumorigenic potential in earlier preclinical studies [28], our study further confirms that mitochondrial reprogramming can indeed elicit beneficial metabolic adaptation potential in human breast cancer cells. From the mechanistic point of view, it has been reported earlier that in hepatocellular carcinoma cells, reactive oxygen species may downregulate catalase expression through the methylation of catalase promoter.[29] We did not test this possibility in our studies but it is plausible that constitutively high reactive oxygen species levels might be the source of reduced catalase expression in the parental 231-P cells. Finally the observation that catalase expression was significantly reduced in human tissue specimens of invasive ductal carcinoma as compared with the normal and hyperplastic breast tissues suggest that development of invasive cancers 
could be causally connected to their propensity to sustain metabolic switch phenotype and/or evade enhancement in mitochondrial function (Supplemental Fig S6). A logical next step will be to develop non-toxic, small molecule probes for modulating mitochondrial complex I and/or antioxidant pathways in a translational setting.

\section{Supplementary Material}

Refer to Web version on PubMed Central for supplementary material.

\section{Acknowledgments}

We gratefully acknowledge financial support from American Cancer Society (RSG-12-144-01-CCE), National Cancer Institute / National Institutes of Health (R21-CA124843), Komen for the Cure foundation (KG090239) and Donna \& Jesse Garber Foundation - all to V.K.R. We also thank Sonal Suhane for her initial help in this project and Dr Bruce Gewertz and Dr Leon Fine for their intramural support and encouragement.

\section{References}

1. Upadhyay M, Samal J, Kandpal M, Singh OV, Vivekanandan P. The Warburg effect: insights from the past decade. Pharmacology \& therapeutics. 2013; 137(3):318-330. DOI: 10.1016/j.pharmthera. 2012.11.003 [PubMed: 23159371]

2. Kim S, Kim do H, Jung WH, Koo JS. Metabolic phenotypes in triple-negative breast cancer. Tumour biology : the journal of the International Society for Oncodevelopmental Biology and Medicine. 2013; 34(3):1699-1712. DOI: 10.1007/s13277-013-0707-1 [PubMed: 23443971]

3. Soga T. Cancer metabolism: Key players in metabolic reprogramming. Cancer science. 2012; doi: $10.1111 /$ cas. 12085

4. Schulze A, Harris AL. How cancer metabolism is tuned for proliferation and vulnerable to disruption. Nature. 2012; 491(7424):364-373. DOI: 10.1038/nature11706 [PubMed: 23151579]

5. Hanahan D, Weinberg RA. Hallmarks of cancer: the next generation. Cell. 2011; 144(5):646-674. DOI: 10.1016/j.cell.2011.02.013 [PubMed: 21376230]

6. Tennant DA, Duran RV, Boulahbel H, Gottlieb E. Metabolic transformation in cancer. Carcinogenesis. 2009; 30(8):1269-1280. [PubMed: 19321800]

7. Deberardinis RJ, Sayed N, Ditsworth D, Thompson CB. Brick by brick: metabolism and tumor cell growth. Current opinion in genetics \& development. 2008; 18(1):54-61. [PubMed: 18387799]

8. Kroemer G. Mitochondria in cancer. Oncogene. 2006; 25(34):4630-4632. [PubMed: 16892077]

9. Warburg O. On respiratory impairment in cancer cells. Science (New York, NY. 1956; 124(3215): 269-270.

10. Vander Heiden MG, Cantley LC, Thompson CB. Understanding the Warburg effect: the metabolic requirements of cell proliferation. Science (New York, NY. 2009; 324(5930):1029-1033.

11. Suhane SKH, Arumugaswami V, Murali R, Ramanujan VK. Mitochondrial NDUFS3 regulates ROS-mediated Onset of Metabolic Switch in Transformed Cells. Biology Open. 2013; 2(2)doi: 10.1242/bio.20133244

12. Santidrian AF, Matsuno-Yagi A, Ritland M, Seo BB, LeBoeuf SE, Gay LJ, Yagi T, FeldingHabermann B. Mitochondrial complex I activity and NAD+/NADH balance regulate breast cancer progression. The Journal of clinical investigation. 2013; 123(3):1068-1081. DOI: 10.1172/ JCI64264 [PubMed: 23426180]

13. Ramanujan VK. Metabolic imaging in multiple time scales. Methods. 2014; 66(2):222-229. DOI: 10.1016/j.ymeth.2013.08.027 [PubMed: 24013043]

14. Suhane S, Ramanujan VK. Thyroid hormone differentially modulates Warburg phenotype in breast cancer cells. Biochemical and biophysical research communications. 2011; 414(1):73-78. DOI: 10.1016/j.bbrc.2011.09.024 [PubMed: 21945435] 
15. Ong SE, Blagoev B, Kratchmarova I, Kristensen DB, Steen H, Pandey A, Mann M. Stable isotope labeling by amino acids in cell culture, SILAC, as a simple and accurate approach to expression proteomics. Molecular \& cellular proteomics : MCP. 2002; 1(5):376-386. [PubMed: 12118079]

16. Wisniewski JR, Zougman A, Nagaraj N, Mann M. Universal sample preparation method for proteome analysis. Nature methods. 2009; 6(5):359-362. DOI: 10.1038/nmeth.1322 [PubMed: 19377485]

17. Cox J, Neuhauser N, Michalski A, Scheltema RA, Olsen JV, Mann M. Andromeda: a peptide search engine integrated into the MaxQuant environment. Journal of proteome research. 2011; 10(4):1794-1805. DOI: 10.1021/pr101065j [PubMed: 21254760]

18. Cox J, Mann M. MaxQuant enables high peptide identification rates, individualized p.p.b.-range mass accuracies and proteome-wide protein quantification. Nature biotechnology. 2008; 26(12): 1367-1372. DOI: $10.1038 /$ nbt.1511

19. Margoliash E, Novogrodsky A. A study of the inhibition of catalase by 3-amino-1:2:4:-triazole. The Biochemical journal. 1958; 68(3):468-475. [PubMed: 13522646]

20. Margoliash E, Novogrodsky A, Schejter A. Irreversible reaction of 3-amino-1:2:4-triazole and related inhibitors with the protein of catalase. The Biochemical journal. 1960; 74:339-348. [PubMed: 13848609]

21. Lisanti MP, Martinez-Outschoorn UE, Lin Z, Pavlides S, Whitaker-Menezes D, Pestell RG, Howell A, Sotgia F. Hydrogen peroxide fuels aging, inflammation, cancer metabolism and metastasis: the seed and soil also needs "fertilizer". Cell cycle. 2011; 10(15):2440-2449. [PubMed: 21734470]

22. Lu H, Forbes RA, Verma A. Hypoxia-inducible factor 1 activation by aerobic glycolysis implicates the Warburg effect in carcinogenesis. The Journal of biological chemistry. 2002; 277(26):2311123115. [PubMed: 11943784]

23. Owens KM, Kulawiec M, Desouki MM, Vanniarajan A, Singh KK. Impaired OXPHOS complex III in breast cancer. PloS one. 2011; 6(8):e23846.doi: 10.1371/journal.pone.0023846 [PubMed: 21901141]

24. Chiavarina B, Martinez-Outschoorn UE, Whitaker-Menezes D, Howell A, Tanowitz HB, Pestell RG, Sotgia F, Lisanti MP. Metabolic reprogramming and two-compartment tumor metabolism: opposing role(s) of HIF1alpha and HIF2alpha in tumor-associated fibroblasts and human breast cancer cells. Cell cycle. 2012; 11(17):3280-3289. DOI: 10.4161/cc.21643 [PubMed: 22894905]

25. Pavlides S, Whitaker-Menezes D, Castello-Cros R, Flomenberg N, Witkiewicz AK, Frank PG, Casimiro MC, Wang C, Fortina P, Addya S, Pestell RG, Martinez-Outschoorn UE, Sotgia F, Lisanti MP. The reverse Warburg effect: aerobic glycolysis in cancer associated fibroblasts and the tumor stroma. Cell cycle. 2009; 8(23):3984-4001. [PubMed: 19923890]

26. Cardaci S, Desideri E, Ciriolo MR. Targeting aerobic glycolysis: 3-bromopyruvate as a promising anticancer drug. Journal of bioenergetics and biomembranes. 2012; 44(1):17-29. DOI: 10.1007/ s10863-012-9422-7 [PubMed: 22328057]

27. Pedersen PL. Warburg, me and Hexokinase 2: Multiple discoveries of key molecular events underlying one of cancers' most common phenotypes, the "Warburg Effect", i.e., elevated glycolysis in the presence of oxygen. Journal of bioenergetics and biomembranes. 2007; 39(3): 211-222. [PubMed: 17879147]

28. Goh J, Enns L, Fatemie S, Hopkins H, Morton J, Pettan-Brewer C, Ladiges W. Mitochondrial targeted catalase suppresses invasive breast cancer in mice. BMC cancer. 2011; 11:191.doi: 10.1186/1471-2407-11-191 [PubMed: 21605372]

29. Min JY, Lim SO, Jung G. Downregulation of catalase by reactive oxygen species via hypermethylation of CpG island II on the catalase promoter. FEBS letters. 2010; 584(11):24272432. DOI: 10.1016/j.febslet.2010.04.048 [PubMed: 20416298] 
a

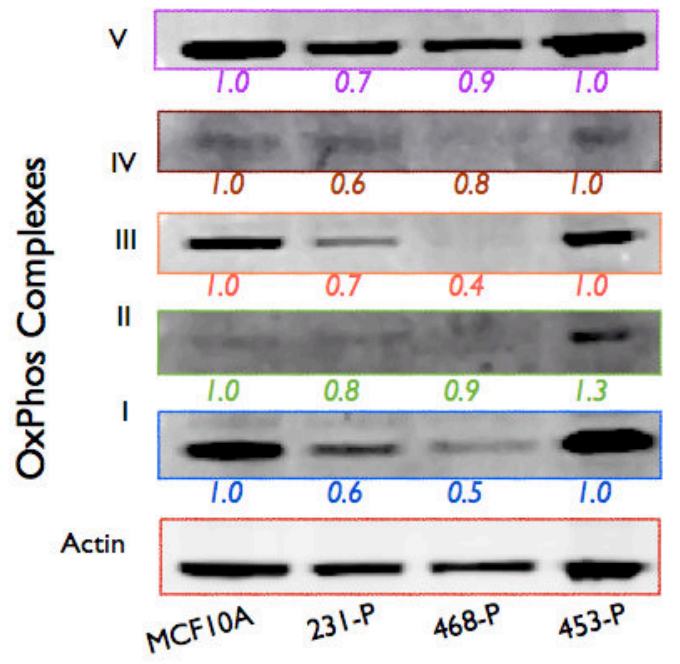

C

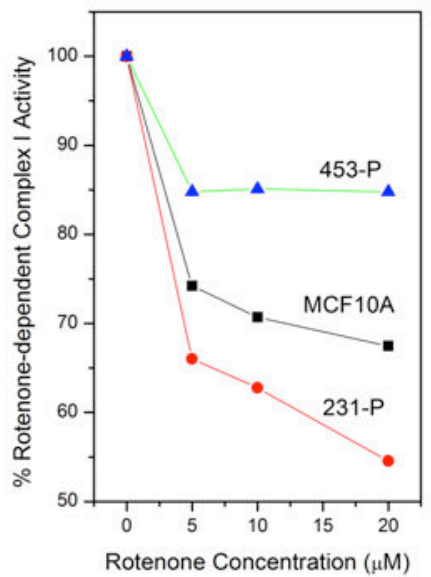

d

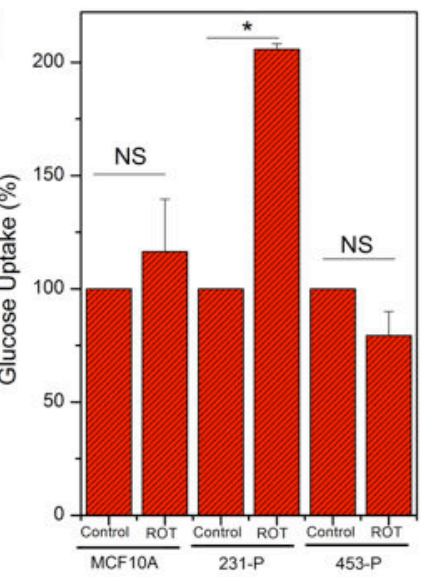

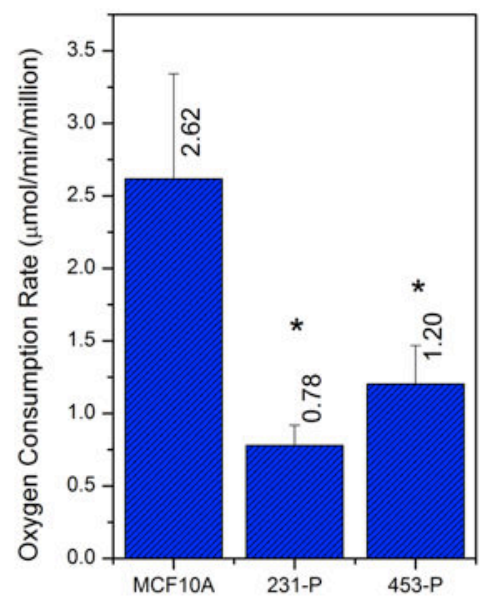

e

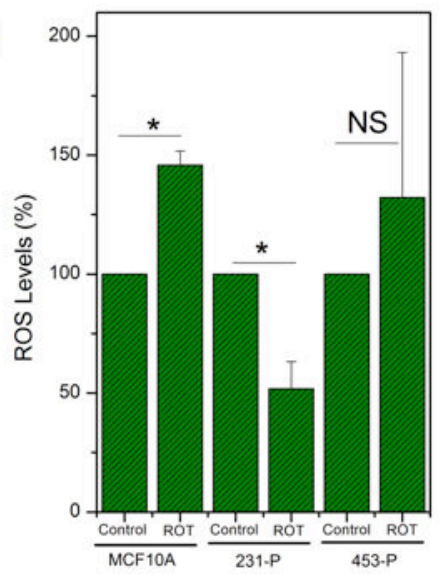

Figure 1.

(a) Representative subunit expression levels of the oxidative phosphorylation (OxPhos) complex in non-transformed mammary epithelial cell line (MCF10A) and three human breast cancer cells. The Mitoprofile OxPhos westernblot cocktail contained a stoichiometric mixture of five mouse monoclonal antibodies specifically targeting the following subunits : complex I subunit NDUFB8 (20 kDa), complex II subunit (30 kDa), complex III subunit Core 2 (47 kDa), complex IV subunit II (24 kDa) and ATP synthase subunit alpha (55 kDa). Densitometric quantitation of band intensities was carried out for each band. Normalized ratio of each band intensity with reference to the loading control (actin band) is denoted below each band in the figure. (b) Oxygen consumption rates (OCR) measured in live cells using the Clark-type oxygen electrode. The data are the mean \pm S.E from 5 independent trials. Comparison of the OCR between the non-transformed MCF10A and the breast cancer cells showed statistical significance ( $\mathrm{p}<0.01)$. (c) Summary of rotenone-dependent mitochondrial complex I activity measured in whole cell lysates. Enzyme activities measured with different rotenone concentration were normalized to the basal activity (without the complex I inhibitor, rotenone) in each cell line. (d) $\&$ (e) FACS results ( $\mathrm{n}=3$ trials) show the mean glucose uptake rates as well as mean free radical generation rates in 
normal and two breast cancer cell lines after acute treatment with 100nM rotenone for 24 hours. Statistical significance $(*)$ denotes $\mathrm{p}<0.05$. 


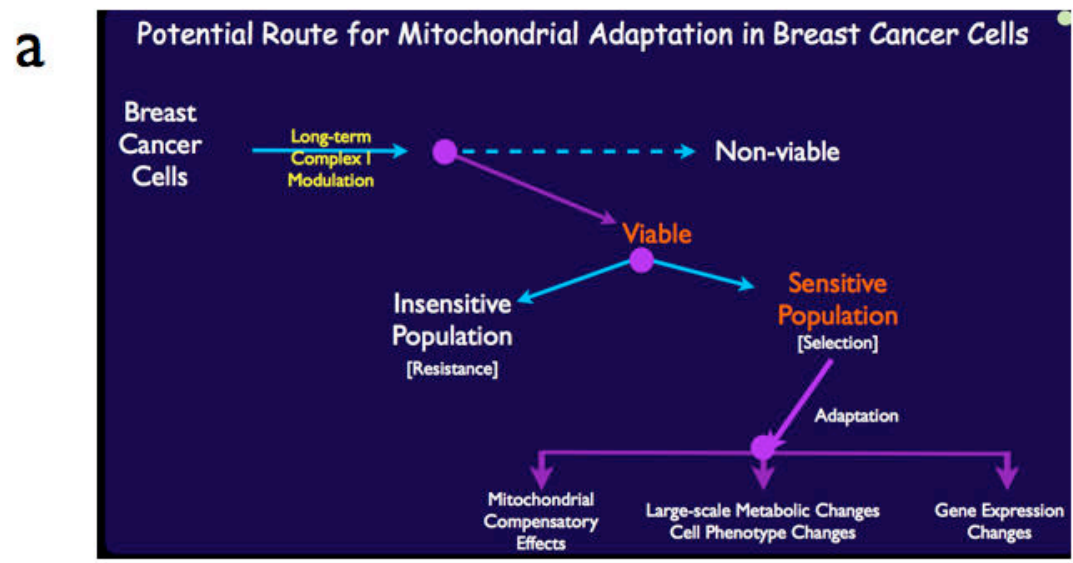

b

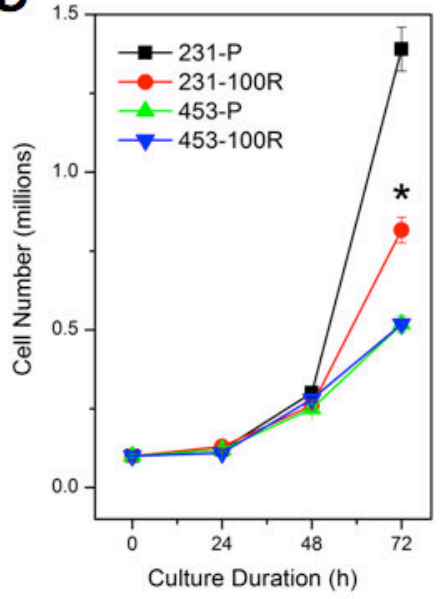

C

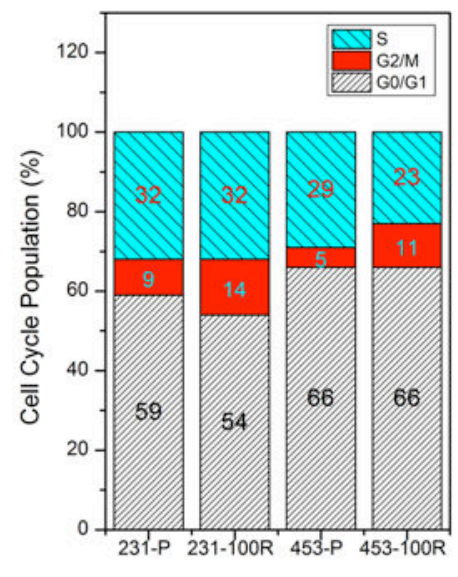

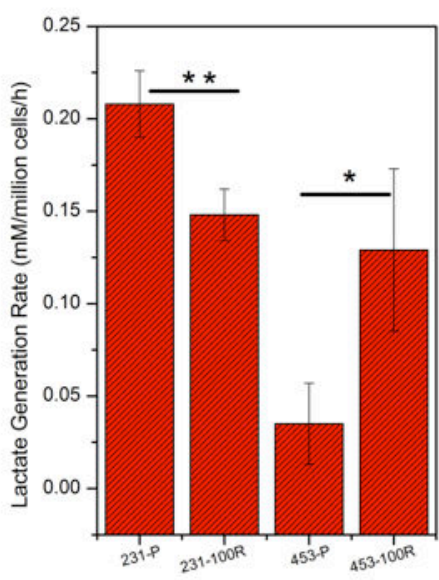

Figure 2.

(a) Schematic of mitochondrial adaptation route in breast cancer cells. The rationale for generating metabolically adapted breast cancer cells stems from the fact that the long term modulation with mitochondrial complex I inhibitor (rotenone) could either render the cells non-viable (due to toxicity) or induce an adaptation route which eventually reprograms the metabolic phenotype in the parental breast cancer cells. More details can be found in the main text. (b \& c) In accordance with the above picture, long-term adaptation to mitochondrial complex I inhibitor (100nM Rotenone) significantly altered the growth characteristics $(\mathrm{p}<0.02$ for $231-\mathrm{P} / 231-100 \mathrm{R}$ cell pair whereas the cell growth rates were not significantly different for the 453-P/453-100R cell pair) and cell cycle parameters in aggressive breast cancer cell line (MDA-MB-231) whereas the non-tumorigenic MDAMB-453 cells did not show any growth reduction. This is in agreement with the rotenonesensitivity as shown in Figure 1(c). Furthermore, the adaptive 231-100R cells showed a significant reduction in lactate generation (a measure of metabolic switch) as compared with the parental 231-P cells whereas the 453-100R cells showed an increase in lactate generation as compared with the parental 453-P cells (d). 
a
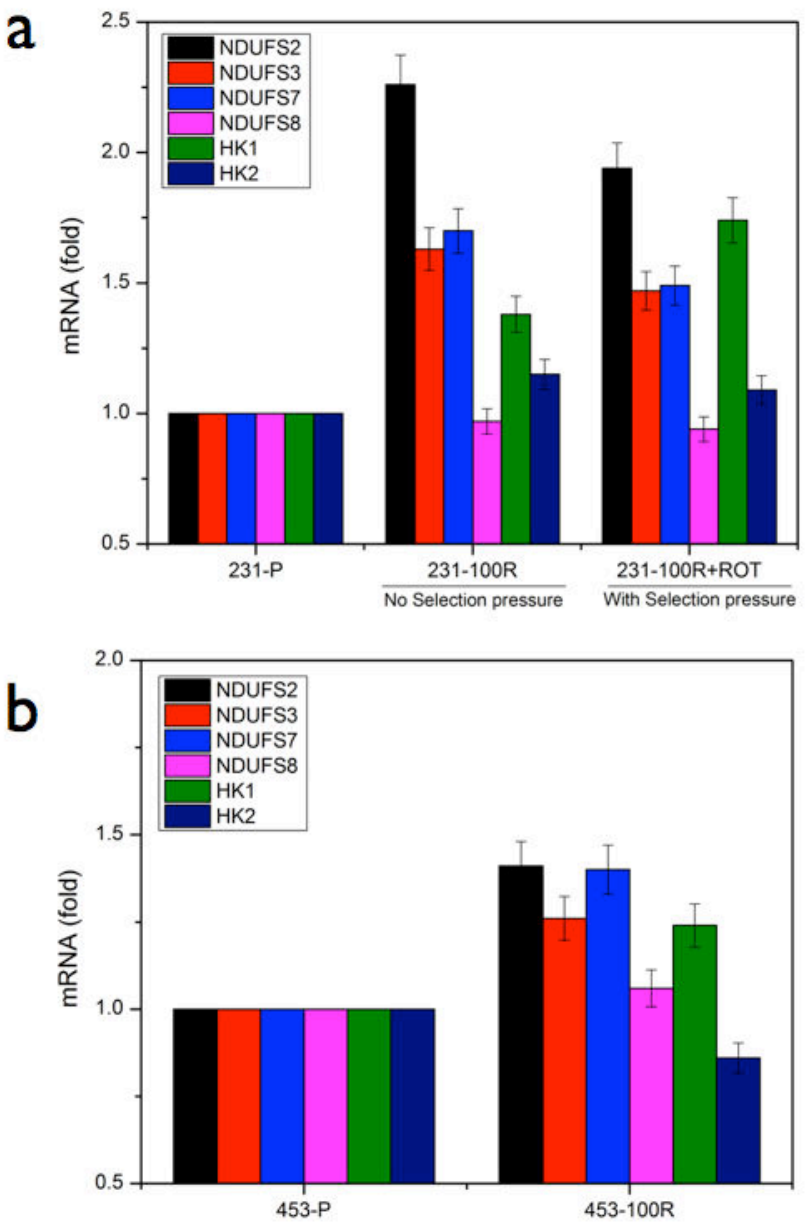

C

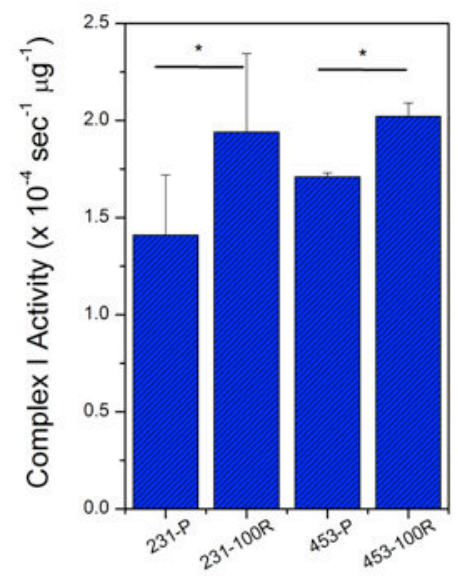

Figure 3.

(a \& b) Real-time qPCR summary of mRNA levels of evolutionarily conserved, core ironsulphur subunits of mitochondrial complex I (NDUFS2/NSUFS3/NDUFS7 \& NDUFS8) genes, as well as two hexokinase isoforms estimated in parental (231-P \& 453-P) and the adaptive (231-100R \& 453-100R) cells. Also shown are the mRNA levels of these representative genes in the adaptive 231-100R cells after maintaining these cells in the selection pressure of $100 \mathrm{nM}$ rotenone to demonstrate that presence of rotenone selection pressure is not a necessary condition for the observed increase in mitochondrial complex I biogenesis. In other words, the adaptation induced mitochondrial biogenesis in 231-100R cells is a more stable phenotype rather than a transient effect. This mitochondrial enhancement at the transcriptional level was further corroborated by functional enhancement in the two breast cancer cell lines as evidenced by significant effects on mitochondrial complex I activity (c) and oxygen consumption rate (d).

Breast Cancer Res Treat. Author manuscript; available in PMC 2016 November 29. 


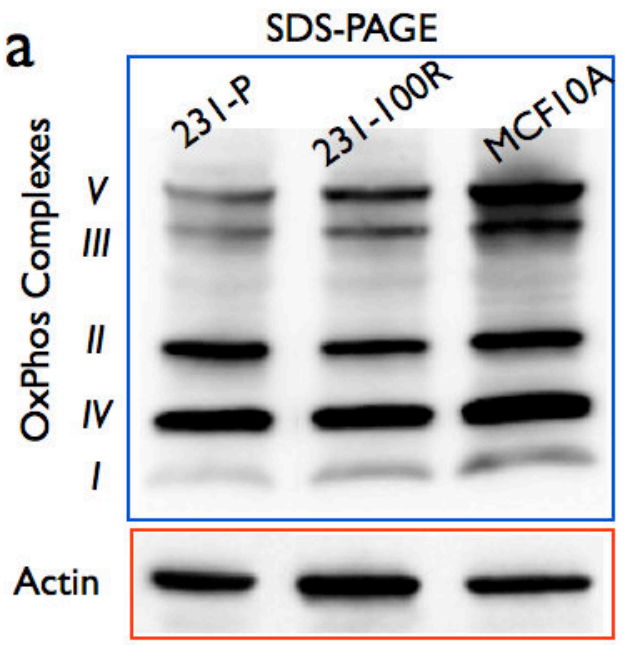

b
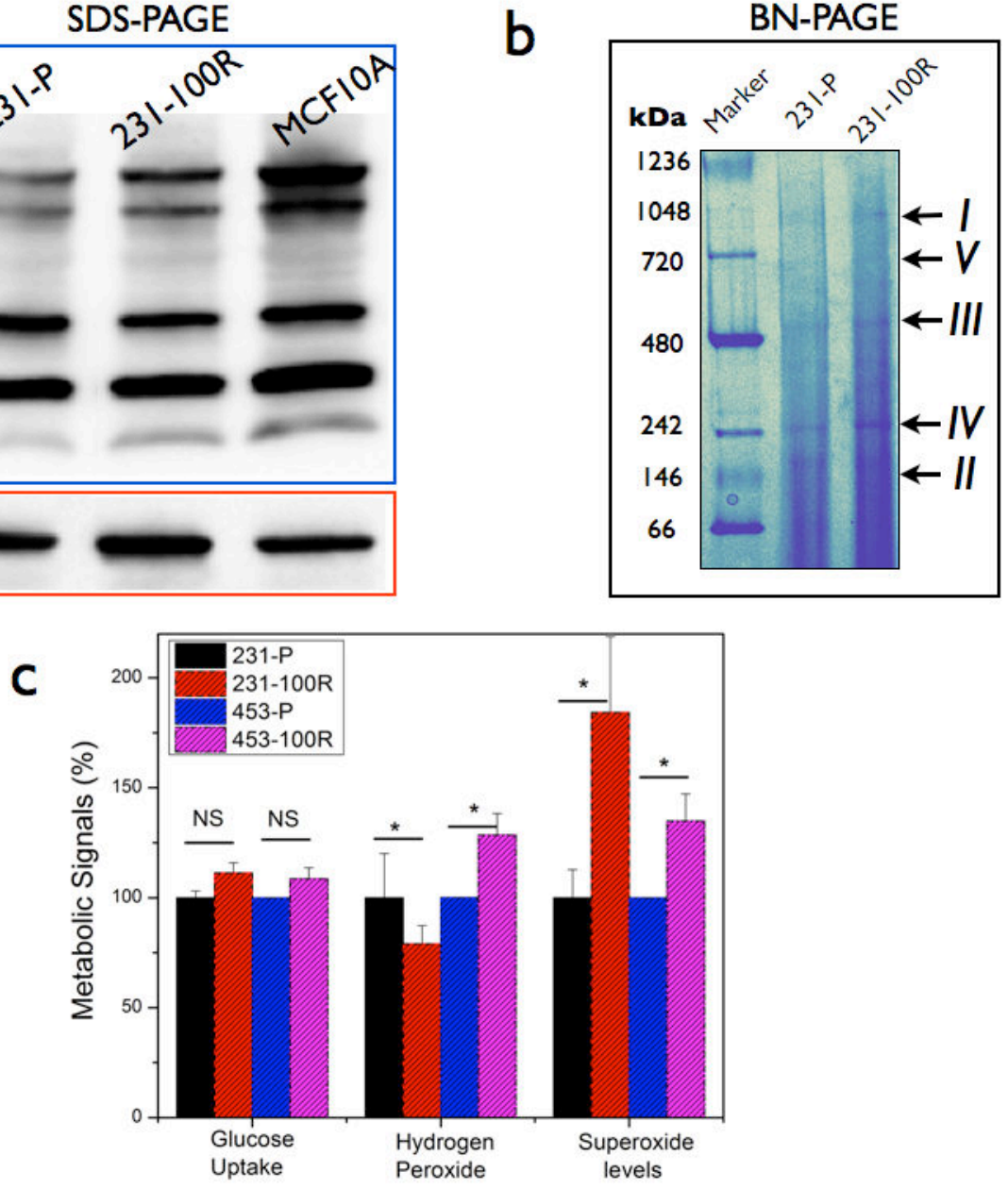

Figure 4.

Adaptation to mitochondrial complex I modulation in MDA231 cells manifested as mitochondrial biogenesis not only at the transcriptional level but also at the protein level. (a) Standard SDS-PAGE-resolved protein bands of representative subunits of mitochondrial electron transport chain (similar to that shown in Figure 1) revealed clear signatures of mitochondrial upregulation in 231-100R cells. Densitometric quantitation of the protein bands normalized to actin band intensity for the three cell lines are as follows: (MCF10A : 21-P : 231-100R) : Complex I (1:0.6:0.7); Complex II (1: 0.8:0.9); Complex III (1: 0.7:0.8); Complex IV (1:0.6:0.6) and Complex V (1: 0.7:0.7) respectively. (b) Representative bluenative (Coomassie) gel electrophoresis results comparing the relative levels of intact (nondenatured) complexes of the electron transport chain. Mitochondrial pellets isolated from fresh cultures of 231-P and 231-100R cells were solubilized in a mixture of sample buffer containing $0.5 \%$ Digitonin. After centrifugation (20,000g for 30 minutes at $4 \mathrm{degC}$ ), $163 \mu \mathrm{g}$ of solubilized mitochondria (non-denatured) was added in each lane in a 4-16\% Bis-Tris gel followed by Coomassie staining and imaging. (c) Mean metabolic signals ( $n=3$ trials) such as glucose uptake rate, hydrogen peroxide generation as well as mitochondrial superoxide levels - measured in live cells by flow cytometry as indicated. 
a 231-P:3D Cultures b

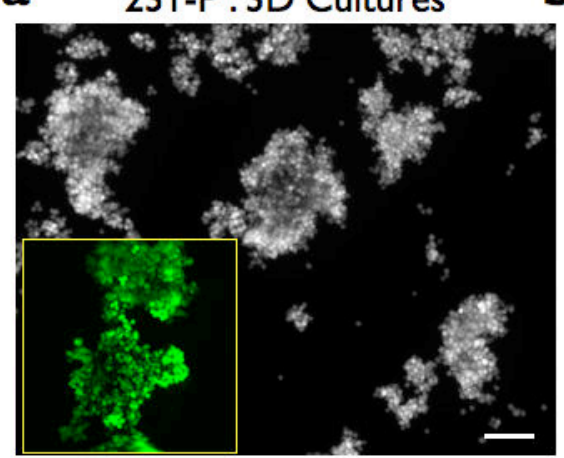

D

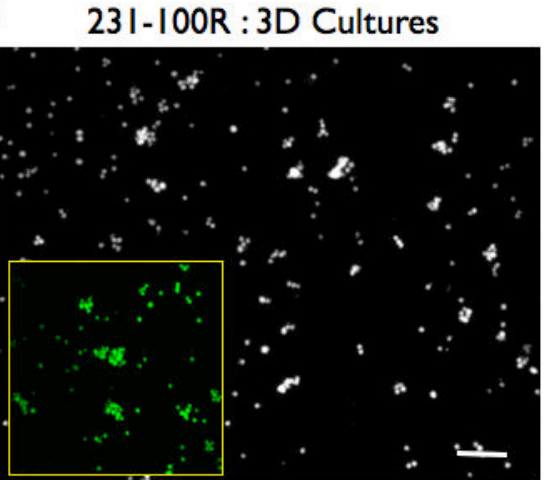

d

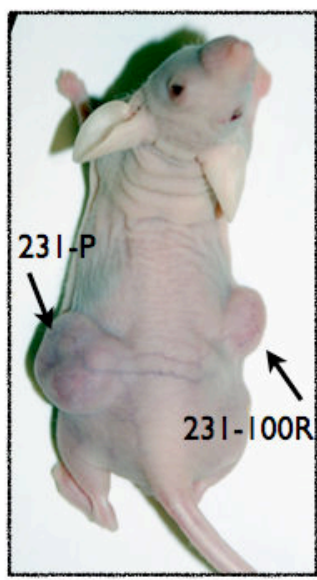

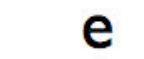

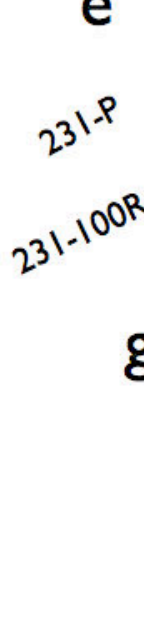

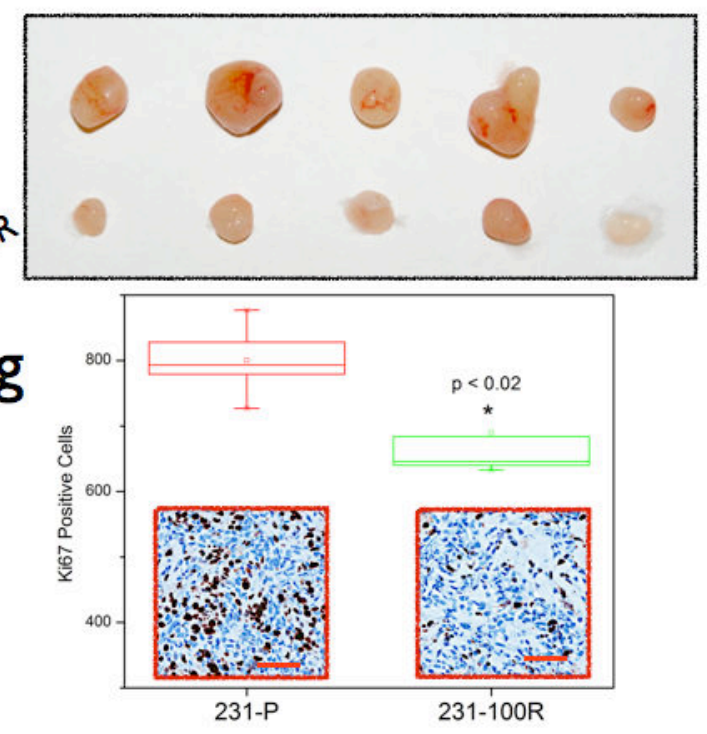
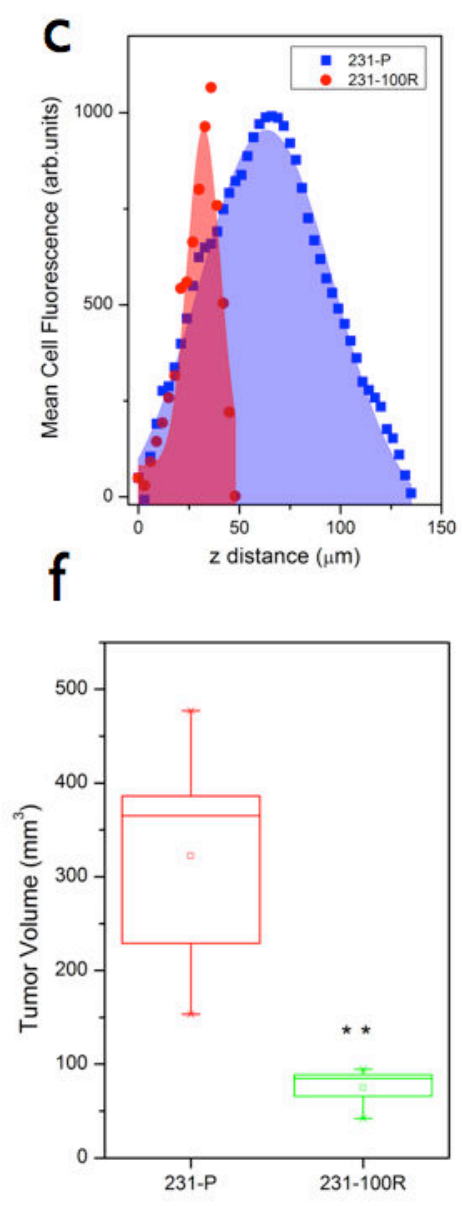

Figure 5.

(a \& b) Representative three-dimensional growth patterns in the parental 231-P and the adaptive 231-100R cells after three weeks of continuous culture in Mammocult medium, an established platform for testing the anchorage-independent growth and potential stem-cell like features in cancer cells. The parental cells were found in larger clusters as compared with the single cells and small cell clusters in the adaptive 231-100R cells. These images were obtained using a stereomicroscope (Nikon AZ100, 2X objective). Scale bar $=100 \mu \mathrm{m}$. The insets in each of these images show the three-dimensional reconstructed area of these cell clusters as measured by a two-photon fluorescence microscope. (c) Three-dimensional growth potential of these cells is demonstrated in the depth profiles where the parental 231-P cells were found to span a depth upto $\sim 150 \mu \mathrm{m}$ whereas the adaptive $231-100 \mathrm{R}$ cells were

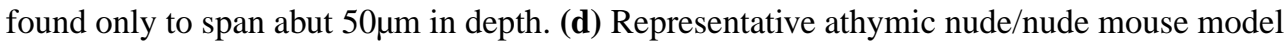
showing the tumor xenografts, 7 weeks after subcutaneous injection with the parental 231-P cells (left flank) and the adaptive 231-100R cells (right flank). (e \& f) Excised tumors from the group of 5 animals showed significant reduction in tumor size/volume in the adaptive 231-100R cells which was also confirmed by immunohistochemistry where the cell proliferation marker, Ki67 was also found be reduced in the tumor xenograft tissue slices obtained from the right flank (231-100R) $(\mathrm{g})$. From a series of 6-8 images from multiple 
fields of view, Ki67-positive cells were counted using ImageJ software. Statistical analysis of the same is shown in $(\mathrm{g})$. 

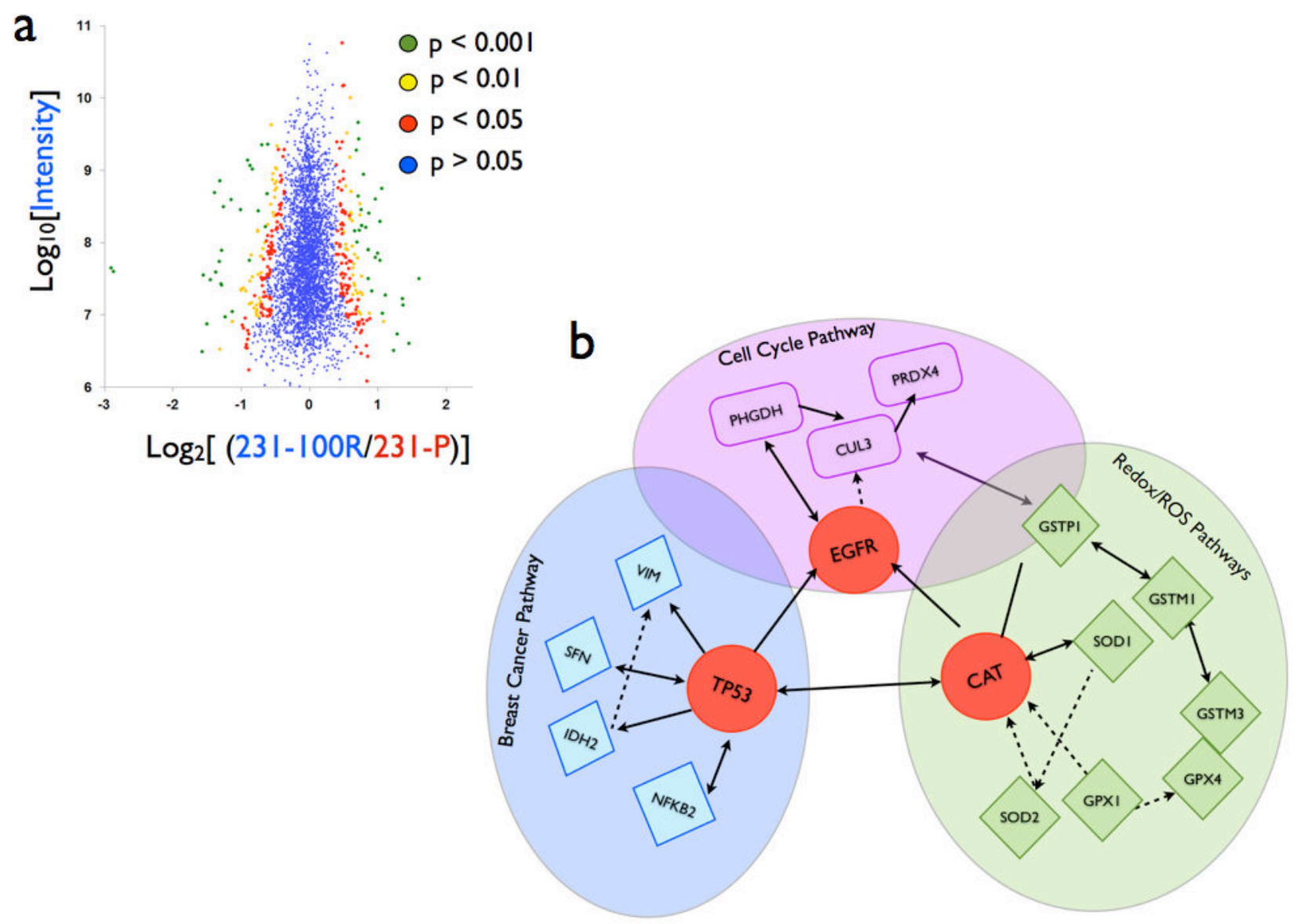

Figure 6.

(a) Statistical significance analysis of differentially expressed proteins in the adaptive 231-100R cells as compared to the parental 231-P cells. The plot shows $\sim 395$ proteins obtained from mass spectrometry based proteomic profiling - which showed statistically significant difference in expression levels between the parental and adaptive cells. (b) Based on the protein expression profiles, an interactogram depicting putative interactions between cell cycle pathway, breast cancer pathway and redox/ROS pathways as obtained from Ingenuity pathway analysis described in the main text and supplemental data. 

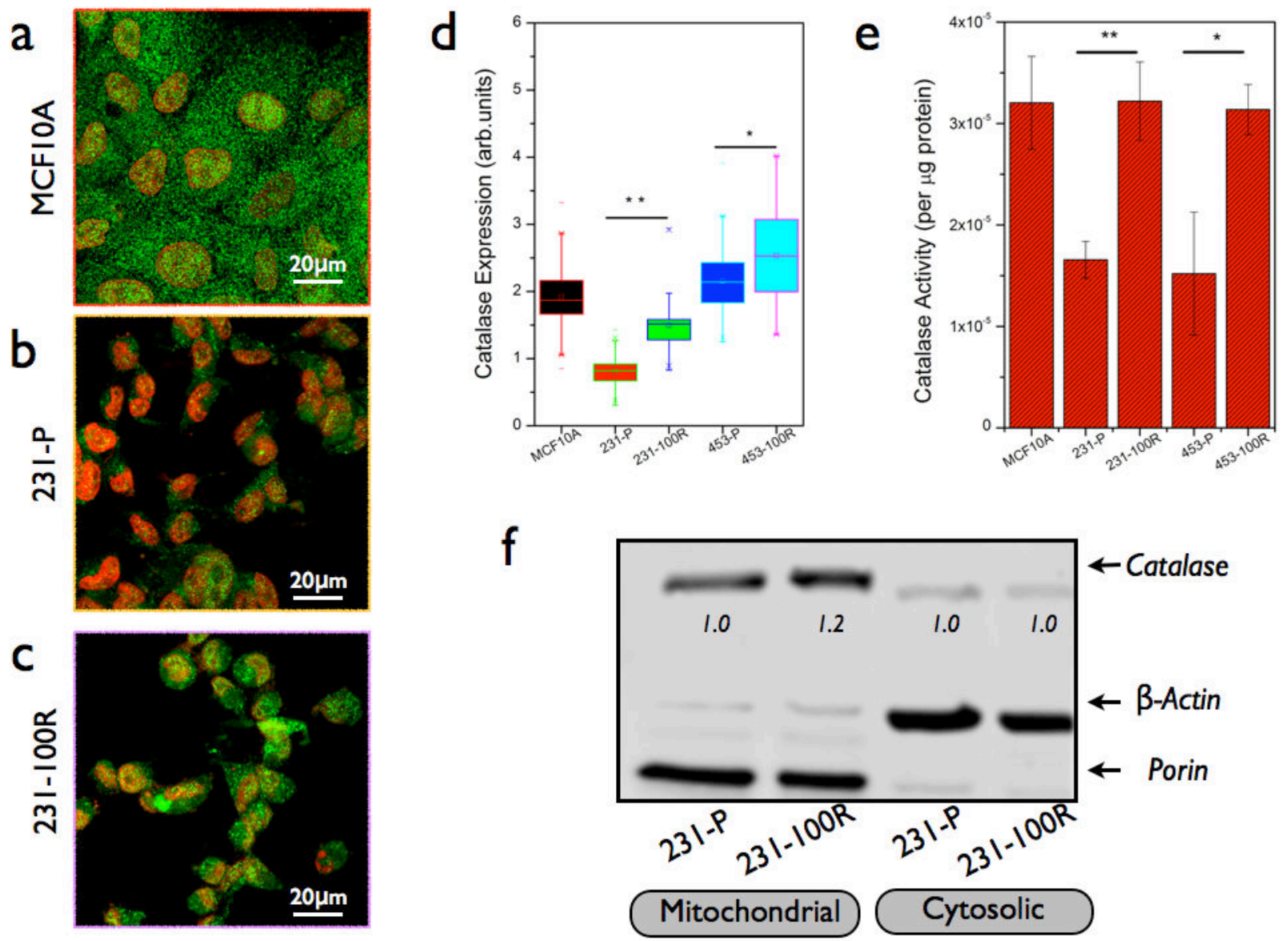

Figure 7.

(a - c) Representative confocal fluorescence images of MCF10A cells, the parental 231-P and the adaptive 231-100R cells probed for catalase expression (green) counterstained with nuclear DAPI (red). (d) Semiquantitative analysis of catalase levels from multiple immunofluorescence images as well as catalase activity measured as described in the main text (e). Immunoblotting further confirmed that mitochondrial catalase was significantly upregulated in the adaptive 231-100R cells as compared with the parental 231-P cells (f). Densitometric quantitation of catalase band intensities (normalized to mitochondrial porin or cytosolic actin) is denoted by the relative ratios below each band. 


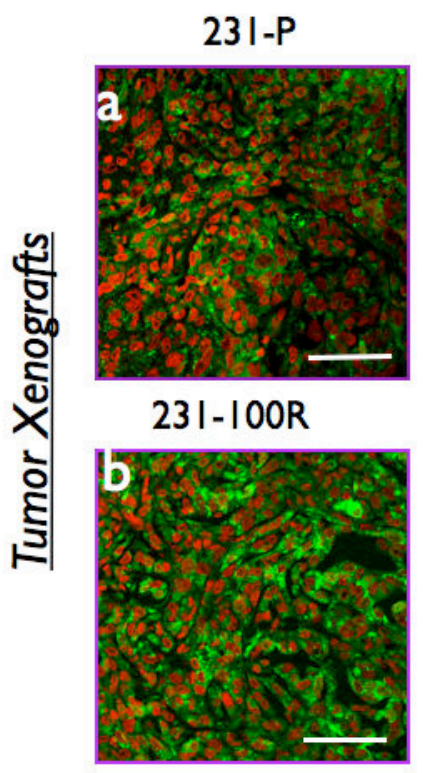

C

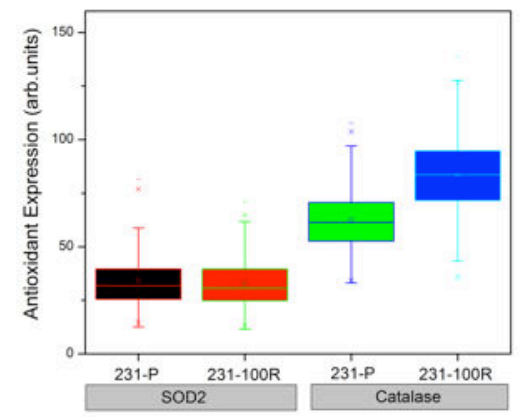

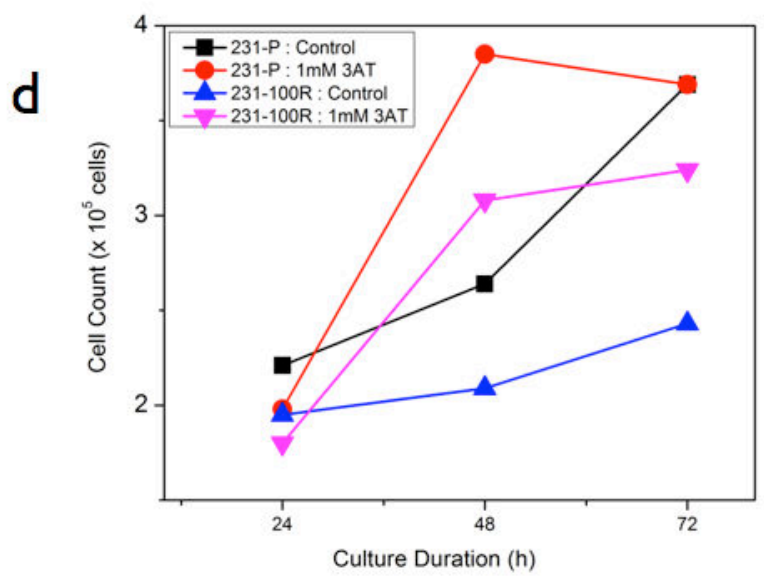

e

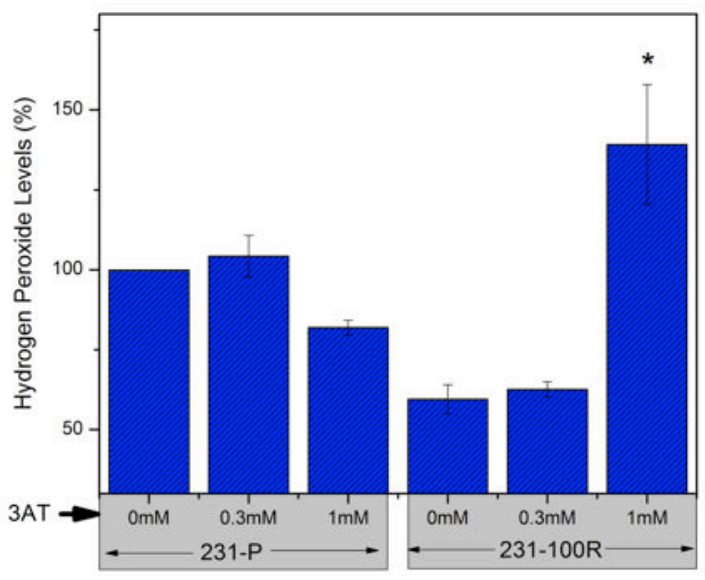

Figure 8.

(a - b) Representative confocal fluorescence images from tumor xenograft tissue sections probed for catalase (green) counterstained with nuclear DAPI (red) and (c) semiquantitative anlaysis of SOD2 and catalase levels from multiple immunofluorescence images of the tumor tissue sections. (d) To test the role of catalase in contributing to reduced cell growth characteristics in the adaptive 231-100R cells, both the parental and adaptive cells were cultured in the presence of a known catalase inhibitor, 3-aminotriazole (3AT) for 48 hours. The two different dosages of 3AT are shown in the figure. 3AT was added every $24 \mathrm{~h}$ in the culture. Inhibition of catalase by $3 \mathrm{AT}$ showed a significant increase in cell proliferation rate in both 231-P and 231-100R cells. (e) Under similar conditions of 3AT treatment, hydrogen peroxide levels were also determined by live cell flow cytometry using a fluorogenic probe, DCFDA that is selective for hydrogen peroxide induced fluorescence. 

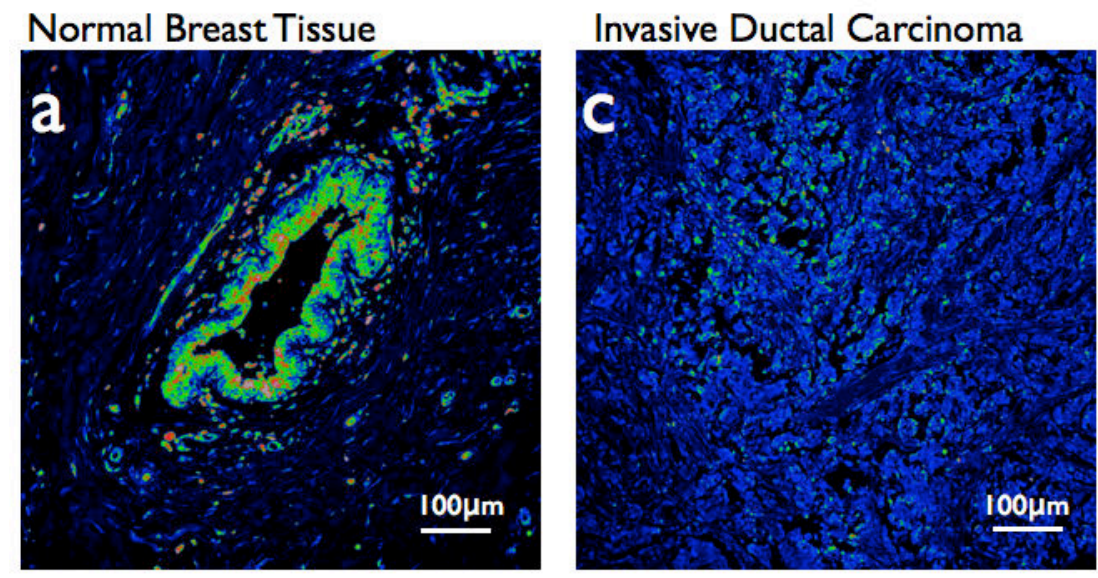

\section{Hyperplasia}
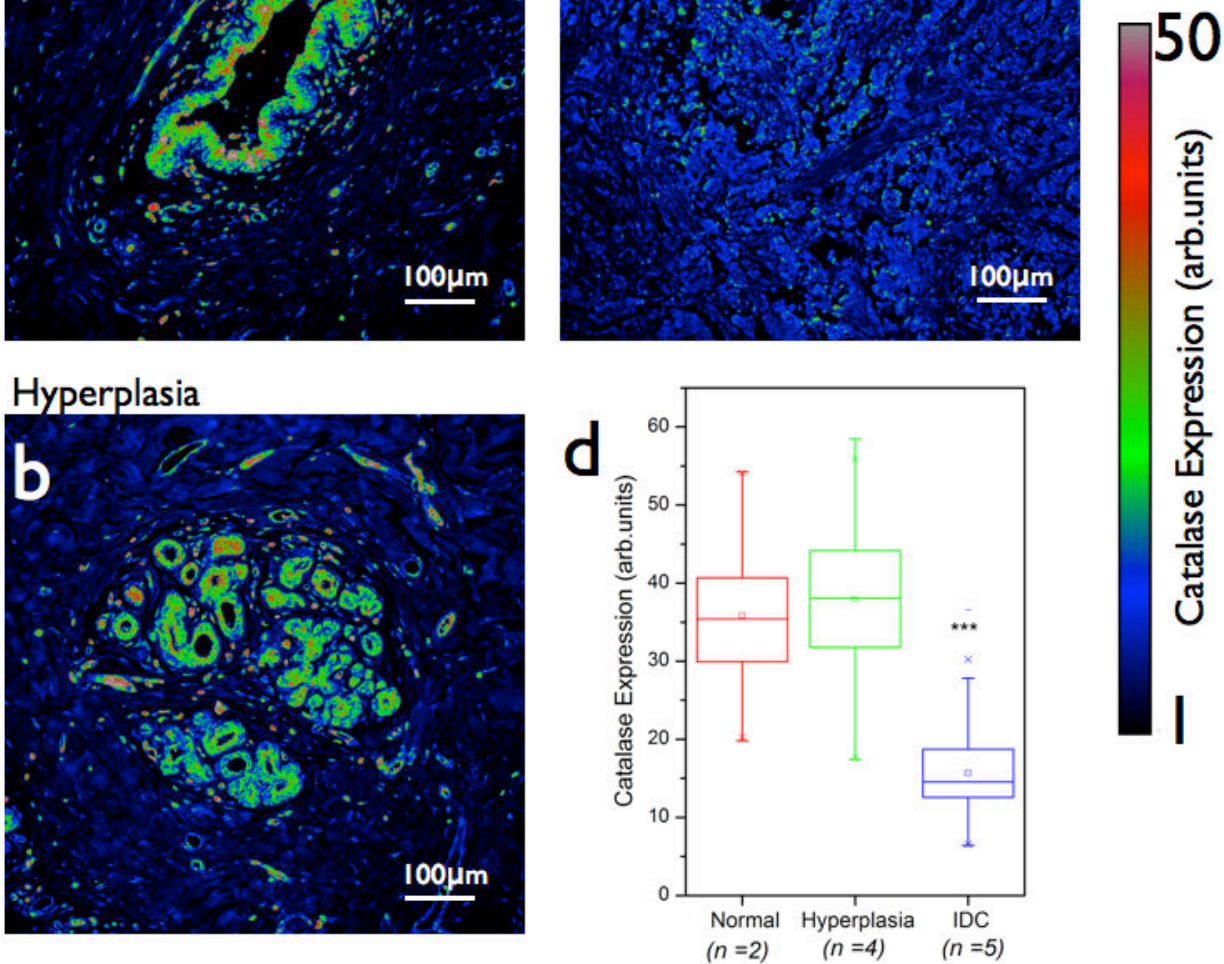

Figure 9.

(a -c) Immunofluorescence images of a human breast cancer tissue microarray probed for catalase expression. Normal breast, hyperplasia and invasive ductal carcinoma patient specimens showed a huge difference in the relative catalase expression levels as summarized in (d). 


\section{Table 1}

Canonical Pathways that show differentially expressed proteomic profiles in the adaptive 231-100R cells as compared with the parental 231-P cells

\begin{tabular}{|c|c|c|c|}
\hline & Pathway Name & $-\log (p$ value $)$ & Molecules Involved in the pathway \\
\hline 1 & $\begin{array}{l}\text { Aryl Hydrocarbon Receptor } \\
\text { Signaling }\end{array}$ & 8.48 & $\begin{array}{l}\text { TP53 (0.67), TRIP11(NAN), GSTM1 }(0.54), \operatorname{SRC}(0.54) \text {, } \\
\text { GSTM3 (0.41), NFKB2 (0.65), CYP1B1 (1.50), TGM2 } \\
(0.61), \text { CTSD (1.44), SP1 (1.47), ALDH3A2 (1.43), } \\
\text { GSTP1 (0.53), MCM7 (0.73), HSPB1 }(0.38) \text {, } \\
\text { GSTK1 (0.67) }\end{array}$ \\
\hline 2 & Protein Kinase A Signaling & 3.62 & $\begin{array}{l}\text { AKAP12 (0.54), MYH10 (0.40), GNAQ (0.50), } \\
\text { PYGB(1.49), PYGL(0.70), NFKB2 (0.65), PRKAG1 } \\
(1.41), \text { TGFBR2 (1.44), MYL9 (1.54), GNAI3 (1.09), } \\
\text { CAMK2D (1.8), FLNC (0.56), PPP1R7 (1.36), } \\
\text { SFN (0.70), GNG12 (0.75), PRKCA (1.39) }\end{array}$ \\
\hline 3 & Apoptosis Signaling & 3.43 & $\begin{array}{l}\text { TP53 (0.67), LMNA (1.58), CAPN2 (1.35), } \\
\text { NFKB2(0.65), RPS6KA1 (0.61), CYCS }(0.74) \text {, } \\
\text { PRKCA (1.39) }\end{array}$ \\
\hline 4 & $\begin{array}{l}\text { Breast Cancer Regulation by } \\
\text { Stathmin1 }\end{array}$ & 3.14 & $\begin{array}{l}\text { TP53 (0.67), GNAI3(1.09), TUBB3(2.02), CAMK2D (1.80), } \\
\text { TUBB6 (0.40), PPP1R7 (1.36), GNAQ (0.50), } \\
\text { GNG12 (0.75), PRKAG1 (1.41), PRKCA (1.39) }\end{array}$ \\
\hline 5 & $\begin{array}{l}\text { NRF2-mediated Oxidative Stress } \\
\text { Response }\end{array}$ & 2.77 & $\begin{array}{l}\text { GSTM1 (0.54), USP14(0.59), SOD1 }(0.70), \text { PPIB }(0.65) \text {, } \\
\text { GSTM3 (0.41), SQSTM1 (0.74), GSTP1 }(0.53) \text {, GSTK1 } \\
(0.67), \text { PRKCA (1.39) }\end{array}$ \\
\hline 6 & 14-3-3-mediated Signaling & 2.7 & $\begin{array}{l}\text { SRC (0.54), TUBB3 (2.02), TUBB6 }(0.40), \text { VIM (1.38), } \\
\text { RPS6KA1 (0.61), SFN }(0.70), \operatorname{PRKCA~}(1.39)\end{array}$ \\
\hline 7 & ERK5 Signaling & 2.56 & $\begin{array}{l}\text { SRC (0.54), RPS6KA3 (0.61), GNAQ }(0.50) \text {, } \\
\text { RPS6KA1 }(0.61), \text { SFN }(0.70)\end{array}$ \\
\hline 8 & Xenobiotic Metabolism Signaling & 2.55 & $\begin{array}{l}\text { GSTM1(0.54), CAMK2D (1.80), GSTM3 }(0.41) \text {, } \\
\text { ALDH3A2 (1.43), SUMO1 (0.67), NFKB2 (0.65) } \\
\text {,GSTP1(0.53),CYP1B1 (1.50), GSTK1 }(0.67) \text {, } \\
\text { PRKCA (1.39), ESD (1.43) }\end{array}$ \\
\hline 9 & TCA Cycle II (Eukaryotic) & 2.37 & SUCLA2 (1.61), IDH3A (0.71), ACO1 (0.67) \\
\hline 10 & Granzyme B Signaling & 1.63 & LMNB2 (1.33), CYCS (0.74) \\
\hline 11 & ERK/MAPK Signaling & 1.62 & $\begin{array}{l}\text { SRC (0.54), PPP1R7 (1.36), ITGA2 (1.43), RPS6KA1 } \\
(0.61), \text { PRKAG1 (1.41), HSPB1 }(0.38), \operatorname{PRKCA~}(1.39)\end{array}$ \\
\hline
\end{tabular}




\begin{tabular}{|c|c|c|c|}
\hline & Pathway Name & $-\log (p$ value $)$ & Molecules Involved in the pathway ${ }^{\#}$ \\
\hline 12 & $\begin{array}{l}\text { Cell Cycle: G2/M DNA Damage } \\
\text { Checkpoint Regulation }\end{array}$ & 1.54 & TP53 (0.67), RPS6KA1 (0.61), SFN (0.70) \\
\hline 13 & p38 MAPK Signaling & 1.48 & $\begin{array}{l}\text { TP53 (0.67), TGFBR2 (1.44), RPS6KA3 (0.67), } \\
\text { RPS6KA1 (0.61), HSPB1 }(0.38) \text {, }\end{array}$ \\
\hline 14 & PI3K/AKT Signaling & 1.41 & $\begin{array}{l}\text { TP53 (0.67), LIMS1(0.55), ITGA2 (1.43), NFKB2 (0.65), } \\
\text { SFN }(0.70)\end{array}$ \\
\hline 15 & Serine Biosynthesis & 1.23 & PHGDH (0.13) \\
\hline
\end{tabular}

\#For each molecule in that specific pathway, the ratio of the protein level in the adaptive 231-100R cells to that in the parental 231-P cells is shown in the parenthesis (blue, italic font) - indicating if that protein is either upregulated (ratio >1) or downregulated (ratio < 1) in 231-100R cells as compared to the parental 231-P cells. 